\title{
Are Biomarkers Predictive of Anthracycline-Induced Cardiac Dysfunction?
}

\author{
Abhidha Malik ${ }^{1 *}$, Pamela Alice Jeyaraj², Rajneesh Calton ${ }^{3}$, Bharti Uppal $^{4}$, Preety \\ Negi$^{2}$, Abhishek Shankar ${ }^{1}$, Jaineet Patil' ${ }^{2}$, Manmohan Kishan Mahajan ${ }^{2}$
}

\begin{abstract}
Background: The early detection of anthracycline- induced cardiotoxicity is very important since it might be useful in prevention of cardiac decompensation. This study was designed with the intent of assessing the usefulness of cardiac troponin T (cTnT) and NT- Pro BNPestimation in early prediction of anthracycline induced cardiotoxicity. Materials and Methods: In this prospective study histologically proven breast cancer patients who were scheduled to receive anthracycline containing combination chemotherapy as a part of multimodality treatment were enrolled. Baseline cardiac evaluation was performed by echocardiography (ECHO) and biomarkers like cardiac troponin $\mathrm{T}$ (cTnT) and $\mathrm{N}$ terminal- pro brain natriuretic peptide (NT- Pro BNP). All patients underwent cTnT and NT- Pro BNP estimation within 24 hours of each cycle of chemotherapy and were followed up after 6 months of initiation of chemotherapy. Any changes in follow up ECHO were compared to ECHO at baseline and cTnT and NT- Pro BNP levels after each cycle of anthracycline-based chemotherapy. Results: Initial data were obtained for 33 patients. Mean change in left ventricular diastolic diameter (LVDD) within 6 months was $0.154 \pm 0.433 \mathrm{cms}$ ( $p$ value=0.049). Seven out of 33 patients had an increase in biomarker cTnT levels (p value=0.5). A significant change in baseline and follow up LVDD was observed in patients with raised cTnT levels ( $p$ value $=\mathbf{0 . 0 2 6}$ ) whereas no change was seen in ejection fraction $(\mathrm{EF})$ and left atrial diameters (LAD) within 6 months of chemotherapy. NT- Pro BNP levels increased in significant number of patients (p value $\leq \mathbf{0 . 0 0 0 1}$ ) but no statistically significant change was observed in the ECHO parameters within 6 months. Conclusions: Functional monitoring is a poorly effective method in early estimation of anthracycline induced cardiac dysfunction. Estimation of biomarkers after chemotherapy may allow stratification of patients in various risk groups, thereby opening window for interventional strategies in order to prevent permanent damage to the myocardium.
\end{abstract}

Keywords: Anthracycline - breast cancer - cardiotoxicity

Asian Pac J Cancer Prev, 17 (4), 2301-2305

\section{Introduction}

Breast cancer is the most frequently diagnosed cancer among women in 140 of 184 countries worldwide. In 2012, 1.7 million women were diagnosed with breast cancer and there were 6.3 million women alive who had been diagnosed with breast cancer in the previous five years. Increase in breast cancer survivors is because of early diagnosis and improved therapies. This increase in survival, however, raises the likelihood that patients will experience side-effects of anticancer therapies (Sawaya et al., 2012).

An overview by Early Breast Cancer 'Trialists' Collaborative Group (EBCTCG) in 2005 reported an absolute survival gain at 5 and 10 years of approximately 3 and $4 \%$ respectively with the inclusion of anthracyclines (compared to non anthracycline) in poly chemotherapy regimens for the management of breast cancer. Since then anthracyclines have been the cornerstone of treatment for women, who require adjuvant chemotherapy for breast cancer.

The most serious effect of repetitive doxorubicin administration is myocardial degeneration causing congestive heart failure which has limited the therapeutic potential of this useful drug. The fact that anthracycline cardiotoxicity is related to cumulative dose suggests that damage starts with the first administration, and additional exposure as far as the heart is concerned is an additive stress (Yau, 2005).

Active monitoring of patients cardiac function can help guide the optimal delivery of chemotherapy, to minimize cumulative cardiotoxicity, while maintaining antitumor activity. Anthracycline induced cardiotoxicity is largely irreversible and it is crucial to detect the myocardial injury 
at its earliest possible stage. A number of techniques are available, including the use of biopsy, imaging modalities, and specific markers of cardiac injury (Frishman et al., 1997). ECG and ECHO are commonly used non-invasive, widely available and low-cost examination for monitoring anthracycline induced cardiotoxicity. The unfortunate limitation of using them to monitor patients during treatment is that a substantial irreversible damage on the cellular level has already occurred by the time cardiac decompensation is appreciated (Steven and Michael, 2009).

The modern advances have brought the selective biochemical markers of cardiac injury into focus. To date, probably the most interesting results have been described with cardiac troponins (Adamcova et al., 2005), other biochemical markers, e.g. natriuretic peptides that have been demonstrated to be useful markers of LV dysfunction in both symptomatic and asymptomatic patients. High concentrations of plasma natriuretic peptides have been described not only in patients with acute myocardial infarction or advanced CHF but also in patients with asymptomatic or minimally symptomatic LV dysfunction (Tsutamoto et al., 1999).

Only a few published reports have evaluated the usefulness of biomarkers in chemotherapy treated patients. The early detection of anthracycline induced cardio toxicity is very important since it might be useful in prevention of cardiac decompensation. This study was designed with the intent of assessing the usefulness of serum cardiac troponinT (cTnT) and NT-pro BNP estimation in the early prediction of anthracycline induced cardiotoxicity.

\section{Materials and Methods}

This study was designed as a prospective study. A total of 36 patients were screened, out of which 33 patients were evaluable.

All patients with histopathological diagnosis of Carcinoma breast with a KPS score of $>80$, normal organ and bone marrow function and normal baseline Echocardiography (ECHO) with no clinical symptoms scheduled to receive anthracycline- based chemotherapy were included in the study. Main exclusion criteria were unwillingness to participate and prior exposure to anthracyclines.

After receiving written informed consent, detailed history, clinical examination, complete cardiac assessment which comprised of baseline, echocardiography, troponin T and NT- Pro BNP estimation by the electrochemiluminescence immunoassay" ECLIA" technique on elecsys and cobas e immunoassay analyzer Roche diagnostics. All patients were assessed by the cardiologist and chemotherapy was administered only after cardiology clearance.

The kind of treatment scheme was based upon the disease status and risk factors. The most common schemes were FAC (5- Fluorouracil, adriamycin and cyclophosphamide), FEC (5- Fluorouracil, epirubicin and cyclophosphamide) or AC followed by P/D (adriamycin followed by paclitaxel or docetaxel) administered once in three weeks after routine complete blood count and biochemistry.

All these patients underwent plasma cTnT and NT Pro BNP estimation within 24 hours of chemotherapy. On follow up after 6 months of initiation of treatment all patients underwent a detailed history, physical examination and a repeat echocardiography (ECHO). Any changes in ECHO at baseline and follow up were correlated with the pattern of rise in CTnT and NT Pro BNP at baseline and after each cycle.

Statistical analyses was performed using the Statistical Package for Social Sciences (SPSS) for Windows version 13. The data was analyzed using the wilcoxon signed rank test and Mc Nemar and student t-test

\section{Results}

We reviewed a total of 36 cases of carcinoma breast treated in the Department of Radiotherapy, Christian Medical College and Hospital, Ludhiana from October 1, 2010 to September 30, 2011, which formed our study group. The demographic data, presenting symptoms, clinical features, histopathological details and treatment details were recorded as per protocol.

Among 33 evaluable patients, the age at presentation ranged between 30 to 70 years. The majority were in the age group between $41-60$ years. Fifteen $(45.4 \%)$ patients had associated co-morbidities, $10(30.3 \%)$ patients had hypertension, $5(15.15 \%)$ patients had diabetes mellitus and $2(6.06 \%)$ patients had both hypertension and diabetes. Twenty (60.6\%) patients had no associated co-morbidities. No statistically significant change was observed in between baseline and follow up ECHO parameters i.e. $\mathrm{EF}, \mathrm{LAD}$ and LVDD in our patients with associated co morbidities.

Adriamycin based regimens were administered in $27(82 \%)$ patients while $6(18 \%)$ patients received epirubicin based chemotherapy. Out of the Twenty seven patients who received adriamycin, $14(51.8 \%)$ patients received $<300 \mathrm{mg} / \mathrm{m}^{2}$ of cumulative dose, while 13 (48.2\%) patients received $\geq 300 \mathrm{mg} / \mathrm{m}^{2}$. The maximum

Table 1. Comparison of Echo Parameters at Baseline and Follow up at 6 Months in Patients with Normal and Raised Ctnt Levels

\begin{tabular}{|c|c|c|c|c|c|c|}
\hline \multirow{2}{*}{$\mathrm{ECHO}$} & \multicolumn{3}{|c|}{$\mathrm{cTnT} \geq \mathrm{ULN}(7)$} & \multicolumn{3}{|c|}{$\mathrm{cTnT} \leq \mathrm{ULN}(26)$} \\
\hline & $\mathrm{EF}$ & LVDD & LAD & $\mathrm{EF}$ & LVDD & LAD \\
\hline Baseline & $56.42 \pm 2.58$ & $4.0014 \pm 0.526$ & $3 \pm 0.27$ & $58.42 \pm 2.98$ & $4.28 \pm 0.335$ & $3.015 \pm 0.34$ \\
\hline Follow up & $57.857 \pm 2.73$ & $3.95 \pm 0.191$ & $2.98 \pm 0.29$ & $58.46 \pm 3.13$ & $4.09 \pm 0.508$ & $3.03 \pm 0.301$ \\
\hline $\mathrm{P}$ value & 0.894 & 0.026 & 0.86 & 0.808 & 0.042 & 0.546 \\
\hline
\end{tabular}


Table 2. Comparison in Echo Parameters at Baseline and Follow up at 6 Months with Patterns Of Rise in Nt Pro Bnp Levels

\begin{tabular}{|c|c|c|c|c|c|c|c|c|c|}
\hline & \multicolumn{3}{|c|}{$\begin{array}{c}\text { Persistent Rise } \\
\mathrm{N}=(3)\end{array}$} & \multicolumn{3}{|c|}{$\begin{array}{c}\text { Fluctuations }>\text { ULN } \\
\mathrm{N}=(14)\end{array}$} & \multicolumn{3}{|c|}{$\begin{array}{l}\text { Fluctuations touching ULN } \\
\mathrm{N}=(15)\end{array}$} \\
\hline ECHO & $\mathrm{EF}$ & LVDD & LAD & $\mathrm{EF}$ & LVDD & LAD & $\mathrm{EF}$ & LVDD & LAD \\
\hline Baseline & $58.3 \pm 3.53$ & $4.53 \pm 0$ & $3.18 \pm 0.21$ & $57.8 \pm 3.73$ & $4.19 \pm 0.39$ & $3.02 \pm 0.28$ & $58.0 \pm 2.56$ & $4.20 \pm 0.42$ & $2.97 \pm 0.37$ \\
\hline Follow up & $60 \pm 7.07$ & $4.5 \pm 0.21$ & $3.11 \pm 0.53$ & $57.9 \pm 2.59$ & $4.07 \pm 0.41$ & $2.98 \pm 0.23$ & $58.7 \pm 3.05$ & $3.97 \pm 0.54$ & $3.04 \pm 0.35$ \\
\hline P value & 0.4 & $<0.0000001$ & 0.28 & 0.2 & 0.85 & 0.46 & 0.52 & 0.35 & 0.83 \\
\hline
\end{tabular}

cumulative dose of epirubicin was $450 \mathrm{mg} / \mathrm{m}^{2}$. The cumulative dose of all 6 patients who received epirubicin based chemotherapy was $\geq 300 \mathrm{mg} / \mathrm{m}^{2}$. No significant change was observed in ECHO parameters in patients receiving $<$ or $\geq 300 \mathrm{mg} / \mathrm{m}^{2}$ of anthracyclines within 6 months of initiation of chemotherapy (see Table1).

Seven $(18.18 \%)$ patients had raised cardiac troponin $\mathrm{T}$ levels at baseline and /or within 24 hours of anthracycline based chemotherapy remaining $26(78.78 \%)$ never showed any increase in cTnT levels. Among the seven patients with raised cTnT levels significant change was seen in baseline and follow up left ventricular diastolic diameter $(p=0.026)$. Whereas in remaining 26 patients who did not have raised cTnT levels during chemotherapy cycles a comparatively less significant change in LVDD was observed at 6 months follow up ECHO (p value=0.042). Other ECHO parameters i.e. EF and LAD in both groups showed no significant change within 6 months of chemotherapy (aee Table 1).

Thirty two out of 33 evaluable patients showed rise in NT- Pro BNP levels atleast once during course of chemotherapy. The Pro BNP levels of 4 (12.1\%) patients, persistently increased with every cycle of anthracycline based chemotherapy. The Pro BNP levels of 14 (42.4\%) patients were observed to fluctuate above the upper limit of normal i.e. $125 \mathrm{pg} / \mathrm{ml}$, while $15(45.4 \%)$ patients showed alternatively increasing and decreasing values of Pro BNP with levels becoming normal sometime during chemotherapy. One patient never showed any abnormal increase in Pro BNP during chemotherapy and was statistically not evaluable. The mean changes in EF, LAD and LVDD in these groups at baseline and during follow up were comparable with no statistically significant difference observed according to pattern of rise in NT Pro BNP levels (see Table 2).

\section{Discussion}

In our study, the patients selected were statistically comparable on the basis of age. The age at presentation ranged from 30 - 64 years with a mean age of 49.42 years. Peak incidence was seen in the age group 40 to 60 years. This is comparable with a cross-sectional study on clinico-morphological patterns of breast cancer, where the average age of the patient at presentation was 47.8 years. The commonest age group of incidence was 45 - 54 years $(31.8 \%)$. Nearly $22 \%$ of cases were below 40 years while $16 \%$ of cases were above the age of 65 years (Saxena et al., 2005). In our study the results are comparable with the study by Willial et al in 2006 where it was observed that breast carcinoma was an etiologically homogenous disease associated with rapidly increasing rates until age 50 years, followed by a slower rate of increase among older women. Therefore, it could be said that age related incidence reaches peak between 4 th- 5 th decade and then declines rapidly.

At diagnosis, a substantial number of women with breast cancer are at significant risk of developing cardiovascular disease due to age and other major cardiac risk factors such as hypertension, diabetes, and obesity, which can adversely affect survival (Yancik et al., 2001). The presence of a cardiac risk factor may increase the chance of a patient experiencing a treatment related cardiac event, as suggested by an early retrospective study (Von hoff et al., 1979). In our study after follow up of 6 months no statistically significant change was observed in the ECHO parameters of patients having associated co morbidities.

Extensive analyses have shown a direct relation between the occurrence of heart failure and the cumulative anthracycline dose. In a study by Suter and Meier in 2002 , it was reported that there is a non-linear correlation between the incidence of contractile dysfunction and the cumulative dose of anthracyclines, and toxicity varies significantly among different anthracyclines. It is difficult to estimate the true incidence of anthracycline induced cardiotoxicity because studies have varied in their definitions, the methods used for measurement of heart function, and the underlying risk of the patient population (Ewer et al., 2004). Data are also primarily retrospective and long-term data are often not available (Elliot, 2006). Early retrospective data by Von hoff et al., 1979, indicate that the incidence of CHF is $3.0 \%$ in patients receiving a cumulative doxorubicin dose of 400 $\mathrm{mg} / \mathrm{m}^{2}$ increasing to $7.5 \%$ at $550 \mathrm{mg} / \mathrm{m}^{2}$ and to $18.0 \%$ at $700 \mathrm{mg} / \mathrm{m}^{2}$. The incidence was found to be lower with a once-weekly schedule compared with a 3 -weekly schedule of doxorubicin administration, suggesting that peak concentrations maybe important in myocardial toxicity. For epirubicin, a large retrospective study reported that the cumulative risk of cardiotoxicity increased from $1.9 \%$ at doses of $800 \mathrm{mg} / \mathrm{m}^{2}$ to $4.3 \%$ at doses of $900 \mathrm{mg} / \mathrm{m}^{2}$ and $15.0 \%$ at $1000 \mathrm{mg} / \mathrm{m}^{2}$ (Ryberg et al., 1998 )

In our study, $81.81 \%$ patients received adriamycin and $18.18 \%$ received epirubicin. In the group of patients receiving adriamycin based chemotherapy $51.8 \%$ patients received cumulative dose of $<300 \mathrm{mg} / \mathrm{m}^{2}$, whereas $48.2 \%$ patients received $\geq 300 \mathrm{mg} / \mathrm{m}^{2}$. The cumulative dose of patients receiving epirubicin was in range of $300-450 \mathrm{mg} /$ $\mathrm{m}^{2}$.

In our study, $45.45 \%$ patients received $<300 \mathrm{mg} / \mathrm{m}^{2}$ cumulative dose of anthracyclines whereas $54.5 \%$ patients 
received cumulative dose of $\geq 300 \mathrm{mg} / \mathrm{m}^{2}$. No significant change in ejection fraction and measurements of left atrial or ventricular diastolic dimension was observed from baseline to follow up ECHO done after 6 months of initiation of anthracyclines. In a study by Nysom et al 1998, depressed left ventricular fractional shortening and left ventricular dilatation were uncommon years after treatment of childhood leukemia when cumulative anthracycline doses were $<300 \mathrm{mg} / \mathrm{m}^{2}$. A threshold of 300 $\mathrm{mg} / \mathrm{m}^{2}$ for late asymptomatic left ventricular dysfunction is supported by subclinical endomyocardial biopsy findings, which are seen at cumulative doses greater than $240 \mathrm{mg} /$ $\mathrm{m}^{2}$ (Billingham and Masek, 1993)

Apple et al., 2007 and Nistico et al., 2007 proposed that Cardiac troponins ( $\mathrm{I}$ and $\mathrm{T}$ ) are sensitive and specific markers of myocardial damage in myocardial infarction and myocarditis. During anthracycline treatment, troponins can be released, and serum levels of cardiac troponin- $\mathrm{T}$ (cTnT) are recognised as biochemical markers of myocardial injury. Experimental studies have shown significant cTnT elevations that could serve as a predictor for anthracycline induced cardiomyopathy whereas clinical studies in this aspect are limited and results are ambiguous because in some studies, administration of anthracyclines did not cause any elevation in cardiac troponins (Horacek et al., 2007) while, cardiac troponins became positive after antharcycline treatment in several other studies by ( Katus et al., 1991, Cardinale et al., 2002, Cardinale et al., 2004). In our study, $78.8 \%$ patients did not have significant increase in cTnT levels within 24 hours after each cycle of anthracycline based chemotherapy ( $p$ value $=0.5)$. In $21.2 \%$ patients an increase in cTnT levels was observed within 24 hours of anthracycline based chemotherapy.

In a study by Kilickap et al. (2005) assessment of cTnT levels and echocardiographic parameters were evaluated 2.5- 11 months after the completion of chemotherapy. No change in LVEF and other systolic and diastolic cardiac function was observed. The investigator suggested, longer follow up of patients who had increased serum levels of cTnT in predicting anthracycline induced cardiotoxicity at an early stage. Lipschultz and colleagues in 1997 have shown that an increased serum level of troponin $\mathrm{T}$ may predict both doxorubicin induced subclinical and clinical cardiotoxicity. They found that elevated serum cTnT was indicative of left ventricular dilatation and wall thinning. However, on the contrary in our study statistically significant decrease in left ventricular diastolic diameter was seen in these patients ( $p$ value $=0.026$ ). The change in ejection fraction and left atrial diameter was not significant.

Clinically apparent cardiac injury can also be determined by natriuretic peptides, which are released from the atria (atrial natriuretic peptide) and the left ventricle brain natriuretic peptide (BNP) in response to circulating volume and intracardiac pressures (Elliot, 2006). In our study, biomarker NT - Pro BNP increased in significant number of patients within 24 hours of chemotherapy with anthracyclines( $\mathrm{p}$ value $\leq 0.0001)$. Anthracycline naïve patients may have higher NT - Pro BNP. In a study by Silver et al., 2004, elevated levels of NT - Pro BNP are most notably associated with CHF, but also with other cardiac conditions. In our study, $15.15 \%$ patients had baseline Pro BNP levels raised above the upper limit of normal i.e $125 \mathrm{pg} / \mathrm{ml}$. No significant change was observed in ECHO parameters of these patients within 6 months of initiation of anthracycline based chemotherapy. In a study by Broeyer et al in 2008, NT - Pro BNP is elevated relatively rapidly after exposure to anthracyclines. This effect was present for each individual subject, during each subsequent chemotherapy cycle. Therefore he assumed that doxorubicin causes sufficient myocardial wall stress or neurohormonal responses on catecholamines or angiotensins to result in acutely elevated concentrations of NT - Pro BNP but this effect takes some time to develop as the NT - Pro BNP concentrations were unchanged immediately after courses $(t=4 h)$. However elevated concentrations were invariably found in each patient at $24 \mathrm{~h}$ time point.

NT- Pro BNP levels in our study, were increasing persistently in $9.09 \%$ patients with every cycle of anthracycline based chemotherapy. The Pro BNP levels of $42.42 \%$ patients were observed to fluctuate above the upper limit of normal i.e. $125 \mathrm{pg} / \mathrm{ml}$, whereas $45.45 \%$ patients showed alternatively increasing and decreasing values of Pro BNP with levels becoming normal after some cycles chemotherapy. One patient never showed any abnormal increase in Pro BNP during chemotherapy and was statistically not evaluable. The mean age at presentation in three groups was comparable with no statistically significant difference. The mean decrease in left ventricular diastolic diameter for patients showing only increase in Pro BNP levels was observed to be spuriously significant ( $\mathrm{p}$ value $\leq 0.0000001$ ).

Our findings are in accordance with a study done by Sandri et al., 2005 who found 3 distinct NT - Pro BNP concentration patterns. Some patients had no change in NT pro BNP concentrations over the 6 sample times, whereas another group had only transient increase with concentration normalizing within $72 \mathrm{~h}$ after end of high dose administration. Only patients with persistent increased NT - pro BNP concentrations that remained increase at $72 \mathrm{~h}$ after end of high dose chemotherapy (HDC) administration developed some form of cardiac impairment during the 12 months of observation with association between worsening of both systolic and diastolic values and persistenly increased NT - pro BNP.

Since long term effects of cardiac sequelae, strongly impacts the quality of life, which is of paramount importance for cancer survivors it is imperative to identify early, the patients with various co-morbid conditions at risk of developing late onset cardiotoxicity. Our study shows that sustained rise in plasma Pro BNP levels as a marker for anthracycline induced cardiotoxicity demonstrate good correlation with echocardiographic findings, whereas a persistently negative troponin test can identify, patients with the lowest risk of cardiotoxicity, who are less likely to encounter cardiac complications, at least within the first 6 months after the initiation of chemotherapy. This determination can lead to a relevant economic impact in oncologic patient management, improving the cost-benefit ratio, because troponin-negative subjects can be safety 
excluded from long-term cardiac monitoring programs, which require high-cost imaging techniques.

To conclude we suggest from this preliminary study that serial measurements of plasma level of Pro BNP and cardiac Troponin T could aid the early prediction of cardiac dysfunction. However, more studies and longer follow up is needed to address the long-term cardiac impact and to develop a more cost-effective clinical tool to assess cardiac risk

\section{References}

Adamcova M, Sterba M, Simunek T et al (2005). Troponin as a marker of myocardiac damage in drug induced cardiotoxicity. Expert opin Drug Saf, 4, 37-46.

Appel JM, Nielsen D, Zerahn B et al (2007). Anthracyclineinduced chronic cardiotoxicity and heart failure. Acta Oncologica, 46, 576-80.

Billingham ME, Masek MA (1993). The pathology of anthracycline cardiotoxicity in children, adolescents and adults. In: Bricker JT, Green DM, D’Angio G (eds) Cardiac toxicity after treatment for childhood cancer. Wiley-Liss, New York; 17-24.

Broeyer FJF, Osanto S, Ritsema vaneck HJ et al (2008). Evaluation of biomarkers for cardiotoxicity of anthracycline based chemotherapy. J Cancer Res Clin Oncol, 134, 961-68.

Cardinale D, Sandri MT, Colombo A et al (2004). Prognostic value of troponin I in cardiac risk stratification of cancer patients undergoing high-dose chemotherapy. Circulation, 109, 2749-54.

Cardinale D, Sandri MT, Martinoni A et al (2002). Myocardial injury revealed by plasma troponin I in breast cancer treated with high-dose chemotherapy. Ann Oncol, 13, 710-15.

Chia S, Bryce C, Gelmon K. Early Breast Cancer Trialist's Collaborative group (EBCTCG) (2005). Effects of chemotherapy and hormonal therapy for early breast cancer on recurrence and 15 year survival: an overview of the randomized trials. Lancet, 365, 1687-1717.

Elliott P (2006). Pathogenesis of cardiotoxicity induced by anthracyclines. Semin Oncol, 33, 2-7.

Ewer MS, Martin FJ, Henderson IC et al (2004). Cardiac safety of liposomal anthracyclines. Semin Oncol, 31, 161-81.

Frishman WH, Sung HM, Yee HC et al (1997). Cardiovascular toxicity with cancer chemotherapy. Curr Probl Cancer, 21, 301-60.

Katus HA, Remppis A, Neumann FJ et al (1991). Diagnostic efficiency of troponin $\mathrm{T}$ measurement in acute myocardial infarction. Circulation, 83, 1360-67.

Kilickap S, Barista I, Akgul E et al (2005). cTnT can be a useful marker for early detection of anthracycline toxicity. Ann Oncol, 16, 798-804.

Nistico C, Bria E, Cuppone F et al (2007). Troponin-T and myoglobin plus echocardiographic evaluation for monitoring early cardiotoxicity of weekly epirubicin-paclitaxel in metastatic breast cancer patients. Anticancer Drugs, 18, 227-32.

Nysom K, Holm K, Lipsitz SR et al (1998). Relationship between cumulative anthracycline dose and late cardiotoxicity in childhood acute lymphoblastic leukemia. J Clin Oncol, 16, 545-50.

Ryberg M, Nielsen D, Skovsgaard T et al (1998). Epirubicin cardiotoxicity: an analysis of 469 patients with metastatic breast cancer. J Clin Oncol, 16, 3502-08.

Sandri MT, Salvatici M, Cardinale D et al (2005). N-terminal pro B- type natriuretic peptide after high dose chemotherapy; marker predictive of cardiac dysfunction? Clin Chem, $\mathbf{5 1}$, 1405-10.

Sawaya H, Sebag IA, Plana JC, et al (2012). Assessment of Echocardiography and Biomarkers for the Extended Prediction of Cardiotoxicity in Patients Treated With Anthracyclines, Taxanes, and Trastuzumab.Circ Cardiovasc Imag, 5, 596-603.

Saxena S, Rekhi B, Bansal A, et al (2005). Clinico-morphological patterns of breast cancer including family history in a New Delhi hospital, India- A cross-sectional study. World J Surg Oncol, 3, 67.

Steven ME and Michael SE (2009). Anthracycline cardiotoxicity: Why are we still interested? Oncol, 23, 3 .

Suter TM and Meier B (2002). Detection of anthracyclineinduced cardiotoxicity: is there light at the end of the tunnel? Ann Oncol, 13, 647-9.

Tsutamoto T, Wada A, Maeda K, et al (1999). Plasma brain natriuretic peptide level as a biochemical marker of morbidity and mortality in patients with asymptomatic or minimal symptomatic left ventricular dysfunction. $E$ Heart $J, 20,1799-80$.

Von Hoff DD, Layard MW, Basa P et al (1979). Risk factors for doxorubicin-induced congestive heart failure. Ann Intern Med, 91, 710-7

Willial FA, Ruth MP, Grace MD et al (2006). Cancer Epidemiology. Biomarkers and Prevent, 15, 1899-905.

Yancik R, Wesley MN, Ries LA et al (2001). Effect of age and comorbidity in postmenopausal breast cancer patients aged 55 years and older. JAMA, 285, 885-92.

Yau TK (2005). Cardiotoxicity after adjuvant anthracyclinebased chemotherapy and radiotherapy for breast cancer. $J$ HK Coll Radiol, 8, 26-9. 